\title{
Mechanism of Reductive Metabolism and Chiral Inversion of Proton Pump Inhibitors ${ }^{\mathrm{S}}$
}

\author{
Chongzhuang Tang, Zhaoqiang Chen, Xiaojian Dai, Weiliang Zhu, Dafang Zhong, \\ and Xiaoyan Chen
}

\begin{abstract}
Shanghai Institute of Materia Medica, Chinese Academy of Sciences, Shanghai, P.R. China (C.T., Z.C., X.D., W.Z., D.Z., X.C.); and University of Chinese Academy of Sciences, Beijing, China (C.T., Z.C., W.Z., D.Z., X.C.)
\end{abstract}

Received December 24, 2018; accepted March 26, 2019

\begin{abstract}
Racemic proton pump inhibitors (PPIs) have been developed into pure enantiomers given superior pharmacokinetic profiles. However, after doses of single enantiomer PPIs, different degrees of chiral inversion were observed. We investigated the relationship between chiral inversion and reductive metabolism of PPIs, as well as the mechanism of reductive metabolism. In liver microsomes and Sprague-Dawley rats, PPI thioethers were stereoselectively oxidized to $(R)$ - and (S)-PPIs, indicating that thioethers could be the intermediates of chiral inversion. By comparing the area under the plasma concentration-time curve ratios of thioether to rabeprazole under different routes of administration and blood sampling site, it was determined that thioether was mainly formed in the liver rather than the intestine. The formation rate of PPI thioethers in liver subcellular fractions was significantly higher than that in buffers.
\end{abstract}

Sulfhydryl-blocking agents, such as $\boldsymbol{N}$-ethylmaleimide, menadione, and ethacrynic acid, inhibited the reductive metabolism of PPIs in vitro, and their corresponding glutathione conjugates were observed. Similar amounts of thioethers were formed in glutathione solutions as in liver subcellular fractions, indicating that biologic reducing agents, instead of reductases, accelerated the reductive metabolism of PPIs. The reduction rates in glutathione solutions were ordered as follows: rabeprazole $>$ omeprazole $>$ lansoprazole $>$ pantoprazole, which was consistent with the natural bond orbital charges of sulfur atoms in these compounds. In conclusion, PPIs were transformed into thioethers by biologic reducing agents in liver, and thioethers continued to be oxidized to two enantiomers, leading to chiral inversion. Furthermore, inhibiting oxidative metabolism of PPIs enhanced reductive metabolism and chiral inversion.

\section{Introduction}

Benzimidazole proton pump inhibitors (PPIs) are used for the treatment of acid-related diseases such as gastric ulcers and nonerosive gastroesophageal reflux disease (Freedberg et al., 2017). Their molecular skeletons contain chiral sulfoxide groups. The early marketed PPIs, including omeprazole (OME), lansoprazole (LAN), pantoprazole (PAN), and rabeprazole (RAB), are all racemic mixtures with two enantiomers exhibiting different pharmacokinetic and/or pharmacodynamic properties (Freedberg et al., 2017). For example, $(R)$-OME and $(R)$-PAN show extensive variability in pharmacokinetics in humans due to CYP2C19 genetic polymorphism, but the metabolic clearance of $(S)$-OME and $(S)$-PAN is independent of CYP2C19 (Ishizaki and Horai, 1999; Horn, 2004). Researchers have gradually realized the therapeutic advantages of single enantiomer forms of PPIs, including reducing the metabolic load on the body, simplifying pharmacokinetics, providing benefit to nonresponders to standard dose of racemate, more

This research was financially supported by the National Natural Science Foundation of China [Grant 81573500] and the Strategic Priority Research Program of the Chinese Academy of Sciences [Grant XDA 12050306].

The authors declare no conflicts of interest.

https://doi.org/10.1124/dmd.118.086090.

S This article has supplemental material available at dmd.aspetjournals.org. homogenous response to treatment, and better efficacy with equal safety (Zhou et al., 2008). Developing single enantiomer PPIs has become a trend (Agranat et al., 2002). (S)-OME, $(R)$-LAN, $(S)$-PAN, and $(R)$-RAB have already been marketed in different countries (Shin and Kim, 2013).

For single enantiomer drugs, good in vitro and in vivo chiral stability is required (Williams et al., 1998). The US Food and Drug Administration states that if a racemate has been marketed and the sponsor wishes to develop the single enantiomer, evaluation should include determination of whether there is significant conversion to the other isomer (Tomaszewski and Rumore, 1994). If the pure enantiomer is chirally unstable, readily converting to a mixture of two enantiomers, then the stereoselective synthesis, chiral separation, and quality control would be in vain. Single enantiomers may undergo enzymatic or nonenzymatic chiral inversion. For example, 2-arylpropionic acid undergoes unidirectional chiral inversion of the inactive $(R)$-enantiomer to the active $(S)$-enantiomer (Ikuta et al., 2017). Thalidomide was marketed in racemic form to treat morning sickness in pregnant women, and strong embryotoxicity and teratogenicity of $(S)$-thalidomide were found. $(R)$-enantiomer was considered to be brought into the market at one point in time, but subsequent studies have proven that the chiral center in thalidomide was unstable in protonated media and underwent rapid chiral inversion. Therefore, $(R)$-thalidomide could still cause toxic reactions due to in vivo chiral inversion (Reist et al., 1998).

ABBREVIATIONS: ABT, 1-aminobenzotriazole; AUC, area under the plasma concentration-time curve; $\mathrm{AUC}_{0-t}$, area under the plasma concentration-time curve from time 0 to the last quantifiable time point; EA, ethacrynic acid; FMO, flavin-containing monooxygenase; GSH, reduced glutathione; HLC, human liver cytosolic (fractions); HLM, human liver microsome; LAN, lansoprazole; LC-MS/MS, liquid chromatographytandem mass spectrometry; MD, menadione; MS, mass spectrometry; $\mathrm{m} / \mathrm{z}$, mass-to-charge ratio; NEM, $\mathrm{N}$-ethylmaleimide; OME, omeprazole; P450, cytochrome P450; PAN, pantoprazole; PPI, proton pump inhibitor; Q-TOF, quadrupole time-of-flight; RAB, rabeprazole; SD, Sprague-Dawley. 


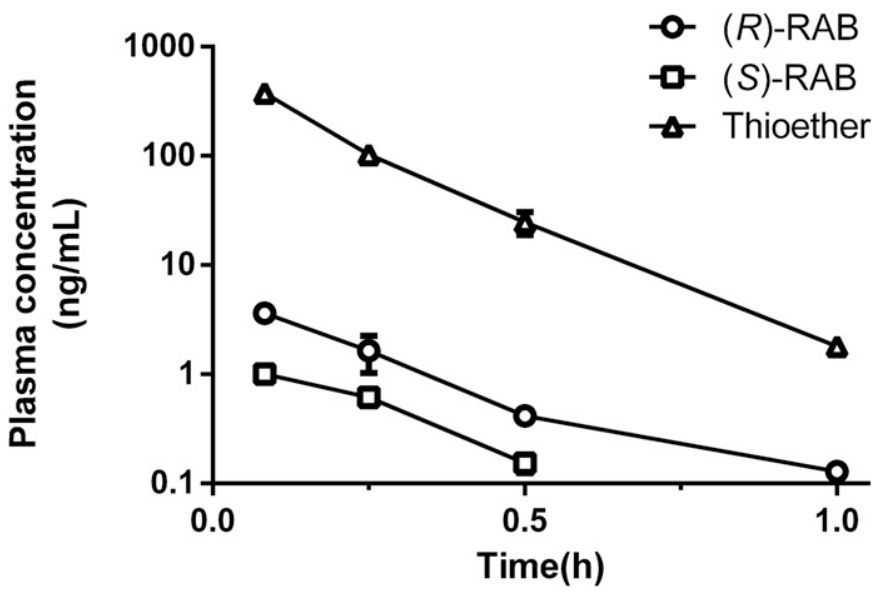

Fig. 1. Average plasma concentration of RAB thioether, $(R)-\mathrm{RAB}$, and $(S)-\mathrm{RAB}$ vs time curves after intravenous administration of RAB thioether $(1 \mathrm{mg} / \mathrm{kg})$ in SD rats. Data are presented as mean \pm S.D. $(n=5)$.

Cases of chiral inversion of single enantiomer PPIs in clinical pharmacokinetic studies have been reported (Andersson et al., 2001; Xie et al., 2004). After oral administration of $40 \mathrm{mg}(S)$-OME, the chiral inversion based on the area under the plasma concentration-time curve (AUC) exposure $\left(\mathrm{AUC}_{R} / \mathrm{AUC}_{S}\right)$ reached $0.4 \%$ in humans. After oral administration of $(R)$-PAN, the average AUC from time 0 to the last quantifiable time point $\left(\mathrm{AUC}_{0-t}\right)$ of $(S)$-PAN accounted for $1.5 \%$ of the total PAN. A phase I clinical trial in our laboratory found that after a single oral dose of $80 \mathrm{mg}$ of $(R)$-anaprazole, the chiral inversion ratio reached $6.3 \% \pm 5.7 \%$ (unpublished data). To date, the mechanism of chiral inversion in vivo and the reasons for the differences in chiral inversion of PPIs have not been reported.

The chemical racemization of sulfoxides occurred at approximately $200^{\circ} \mathrm{C}$ through pyramidal inversion (Rayner et al., 1968; Marom et al., 2007); therefore, the sulfoxide groups were considered chirally stable in vivo. We speculated that the chiral inversion of PPIs was related to their metabolic transformation. Oxidative metabolism and reductive metabolism of PPIs could both occur in vivo to form sulfone and thioether, respectively. According to our early results, PPI sulfone could not be remetabolized to sulfoxide, whereas thioethers could be easily oxidized to sulfoxides. Consequently, a hypothesis was proposed that thioether metabolites of PPIs were the intermediate of chiral inversion. In this study, we investigated the relationship between chiral inversion and reductive metabolism of PPIs, as well as the mechanism of reductive metabolism.

\section{Materials and Methods}

Chemicals and Reagents. Pooled human liver microsomes (HLMs), human liver cytosolic (HLC) fractions, and recombinant flavin-containing monooxygenases
[(FMOs), including FMO1, FMO3, and FMO5] were supplied by BD Gentest (Woburn, MA). Fresh rat liver homogenate and rat liver S9 were prepared according to the reported methods (Esterbauer et al., 1985; Bourland et al., 1998). (R)-LAN, $(S)$-LAN, $(S)$-OME, and $(R)$-PAN were purchased from TRC Chemicals (Toronto, Canada). (S)-PAN was bought from Sun-Wise Pharmaceutical (Hefei, China). (R)-OME was purchased from Santa Cruz Biotechnology (Santa Cruz, CA). $(R)-\mathrm{RAB}$ and $(S)$-RAB were kindly provided by Aosaikang Pharmaceutical (Jiangsu, China). The racemic forms of OME, LAN, PAN, and RAB and their thioether metabolites, $(R)$-LAN-d4, 1-aminobenzotriazole (ABT), reduced glutathione (GSH), NADPH, NADH, ethacrynic acid (EA), menadione (MD), $N$-ethylmaleimide (NEM), cysteine, and DMSO, were purchased from Meilunbio (Dalian, China).

Animal Experiments. All procedures in the animal studies were performed in accordance with the Guide for the Care and Use of Laboratory Animals of Shanghai Institute of Materia Medica, Chinese Academy of Sciences. Male Sprague-Dawley (SD) rats weighing 180-220 g were acclimatized for at least 7 days. Portal vein-cannulated male SD rats were purchased from Vital River Laboratory Animal Technology Co., Ltd. (Beijing, China). Animals were fasted for 12 hours with free access to water before the experiments. Blood samples were collected predosage (0 hour) and $0.083,0.25,0.5,1,1.5,2,3$, and 4 hours postdosage in tubes containing heparin; additionally, two blood samples were also collected at 6 and 8 hours postdosage in the experiment to investigate the effect of inhibiting cytochrome P450 (P450) enzymes on chiral inversion. Plasma was acquired after centrifugation of blood samples at $14,000 \mathrm{rpm}$ for 5 minutes. The isolated plasma samples $(50 \mu \mathrm{l})$ were placed in covered storage tubes containing $1 \%$ diethylamine solution $(2 \mu \mathrm{l})$ as a stabilizer (Uno et al., 2005) and stored at $-80^{\circ} \mathrm{C}$ until analysis.

Biotransformation of RAB Thioether in SD Rats. RAB thioether was dissolved in DMSO and then diluted with normal saline to $200 \mu \mathrm{g} / \mathrm{ml}$. The final concentration of DMSO was $1 \%$. RAB thioether was administered intravenously via the tail vein to five male SD rats at a dose of $1 \mathrm{mg} / \mathrm{kg}$. Plasma samples were collected, prepared, and stored as mentioned previously.

Pharmacokinetic Study of $(R)$-RAB in SD Rats via Various Routes of Administration and Blood Sampling Site. This experiment aimed to determine whether the intestine was the main site of PPI reductive metabolism. The portal vein-cannulated SD rats $(n=5)$ were orally administered $3 \mathrm{mg} / \mathrm{kg}(R)-\mathrm{RAB}$. The normal male SD rats were divided into two groups $(n=4$ in each) and administered the same dose of $(R)-\mathrm{RAB}$ via intravenous and portal vein administration. Rats in the portal vein administration group were anesthetized by intraperitoneal injection of Nembutal $(30 \mathrm{mg} / \mathrm{kg})$, and then the hepatic portal vein was exposed by abdominal incision for administration. $(R)$-RAB was dissolved in sodium bicarbonate buffer $(\mathrm{pH} 10)$. In the portal vein-cannulated rats, blood samples were taken from the portal vein and retro-orbital venous plexus at the same time. In the other two groups, blood samples were collected only from the retro-orbital venous plexus.

Effect of Inhibiting P450 Enzymes on Chiral Inversion. Rats were divided into the ABT group $(n=4)$ and control group $(n=4)$. In the ABT group, rats were orally administered $100 \mathrm{mg} / \mathrm{kg}$ of ABT 16 hours prior to the experiment, and the rats in the control group were given the same volume of normal saline. Then, rats were intravenously injected with $3 \mathrm{mg} / \mathrm{kg}$ of $(R)-\mathrm{RAB}$. Plasma samples were collected, stored, and prepared as mentioned previously.

Incubation of RAB in Liver Subcellular Fractions. RAB was dissolved in DMSO to obtain stock solutions $(100 \mathrm{mM})$, which were then diluted with PBS solution to the desired concentration, and the final system contained a DMSO

TABLE 1

Kinetic parameters for the formation of $(R)$ - and $(S)$-PPIs from PPI thioethers in HLMs

Values are mean \pm S.D., $\mathrm{n}=3$

\begin{tabular}{|c|c|c|c|c|c|c|c|c|}
\hline \multirow{2}{*}{ Parameter } & \multicolumn{2}{|c|}{ OME } & \multicolumn{2}{|c|}{ LAN } & \multicolumn{2}{|c|}{ PAN } & \multicolumn{2}{|c|}{ RAB } \\
\hline & $R$ & $s$ & $R$ & $S$ & $R$ & $s$ & $R$ & $s$ \\
\hline $\begin{array}{l}V_{\max }(\mathrm{nmol} / \mathrm{min} \text { per } \\
\text { milligram protein })\end{array}$ & $1.1 \pm 0.0$ & $0.95 \pm 0.03$ & $0.12 \pm 0.01$ & $0.032 \pm 0.002$ & $0.051 \pm 0.002$ & $0.16 \pm 0.01$ & $0.27 \pm 0.01$ & $0.18 \pm 0.01$ \\
\hline$K_{\mathrm{m}}(\mu \mathrm{M})$ & $36 \pm 3$ & $74 \pm 6$ & $77 \pm 14$ & $78 \pm 13$ & $6.1 \pm 1.2$ & $11 \pm 1.8$ & $18 \pm 1$ & $72 \pm 4$ \\
\hline $\begin{array}{l}V_{\max } / K_{\mathrm{m}}(\mu \mathrm{l} / \mathrm{min} \text { per } \\
\text { milligram protein })\end{array}$ & $31 \pm 2$ & $13 \pm 1$ & $1.7 \pm 0.2$ & $0.41 \pm 0.04$ & $8.4 \pm 1.4$ & $14 \pm 2$ & $15 \pm 1$ & $2.5 \pm 0.1$ \\
\hline
\end{tabular}


TABLE 2

AUCs of the RAB thioether and $(R)-\mathrm{RAB}$ after three administration routes of $3 \mathrm{mg} /$ $\mathrm{kg}$ of $(R)-\mathrm{RAB}$

The administration routes included oral administration $(\mathrm{n}=5)$ in portal vein-cannulated rats, portal vein administration $(\mathrm{n}=4)$, and intravenous administration $(\mathrm{n}=4)$ in normal $S D$ rats. Results are expressed as mean \pm S.D.

\begin{tabular}{lccc}
\hline Administration Route & $\begin{array}{c}\mathrm{AUC}_{0-t} \text { of Thioether } \\
(\mathrm{ng} \cdot \mathrm{h} / \mathrm{ml})\end{array}$ & $\begin{array}{c}\mathrm{AUC}_{0-t} \text { of }(R)-\mathrm{RAB} \\
(\mathrm{ng} \cdot \mathrm{h} / \mathrm{ml})\end{array}$ & AUC Ratio $^{a}$ \\
\hline & & & $\%$ \\
$\mathrm{Oral}^{b}$ & $3.75 \pm 1.09$ & $12.8 \pm 5.3$ & 30.6 \\
$\mathrm{Oral}^{c}$ & $15.5 \pm 3.0$ & $137 \pm 64$ & 11.8 \\
Portal vein & $25.0 \pm 3.38$ & $91.3 \pm 34.2$ & 28.6 \\
Intravenous & $73.9 \pm 8.75$ & $500 \pm 46.7$ & 15.5 \\
\hline
\end{tabular}

${ }^{a} \mathrm{M}_{\mathrm{RAB}} \times \mathrm{AUC}_{0-t \text { thioether }} / \mathrm{M}_{\text {thioether }} \times \mathrm{AUC}_{0-t \mathrm{RAB}}$, where $\mathrm{M}$ represents the average molecular weight.

${ }^{b}$ From the systemic plasma concentration in circulation

${ }^{c}$ From the portal vein plasma concentration.

concentration of $0.1 \%(\mathrm{v} / \mathrm{v})$. RAB $(1.5 \mu \mathrm{M})$ was incubated in several kinds of liver subcellular components [HLMs, HLC fractions, heated HLC fractions, freshly prepared rat liver homogenate, and acetonitrile extract of freshly prepared rat liver homogenates $(5: 1, \mathrm{v} / \mathrm{v})]$ at $37^{\circ} \mathrm{C}$ for 1 hour. Reduced coenzymes such as NADPH $(1 \mathrm{mM})$ and NADH $(0.1 \mathrm{mM})$ were added to the HLC fraction incubations to evaluate whether reductases were involved in the reductive metabolism. $(R)$ - RAB $(10 \mu \mathrm{M})$ was also incubated in rat liver $\mathrm{S} 9(1 \mathrm{mg} / \mathrm{ml})$ in the absence $(-)$ and presence $(+)$ of NADPH $(1 \mathrm{mM})$ or GSH $(1 \mathrm{mM})$, either individually or in combination. After incubation, $100 \mu \mathrm{l}$ of ice-cold acetonitrile containing $0.1 \%$ diethylamine was added to terminate the reaction, and the mixture was stored at $-80^{\circ} \mathrm{C}$ until the analysis of $\mathrm{RAB}$ thioether. The results are presented as the mean \pm S.D. from three triplicate experiments. The main metabolites in the rat liver S9 incubations were detected using ultra-performance liquid chromatography/quadrupole time-of-flight (Q-TOF) mass spectrometry (MS).

Inhibition of the Reductive Metabolism of RAB. HLC fractions were preincubated with different concentrations $(25,100$, and $1000 \mu \mathrm{M})$ of sulfhydrylblocking agents, such as NEM, EA, and MD, for 15 minutes. Then, RAB was added to initiate the reaction. After 1-hour incubation, ice-cold acetonitrile containing $0.1 \%$ diethylamine was added to terminate the reaction, and stored at $-80^{\circ} \mathrm{C}$ until analysis of RAB thioether.

Incubation of PPIs in GSH and Cysteine Solutions. Four PPIs were incubated with GSH to confirm whether the thiol-containing molecules in liver subcellular fractions could accelerate the reduction of PPIs and to evaluate the effect of thiol concentration on the reduction of PPIs. GSH and cysteine were prepared in deionized water to obtain $10 \mathrm{mM}$ stock solutions, which were then gradually diluted to different concentrations $(5,10,25,50,100,500$, and $1000 \mu \mathrm{M}$ ) with PBS or potassium phosphate (a stronger capacity buffer used to avoid a change in acidity due to increased GSH or cysteine concentration). RAB $(1.5 \mu \mathrm{M})$ was incubated with GSH or cysteine solutions for 1 hour at $37^{\circ} \mathrm{C}$. After incubation, the reaction was stopped with cold acetonitrile solution containing $1 \mathrm{mM}$ NEM. Equal concentrations of OME, LAN, PAN, and RAB $(1.5 \mu \mathrm{M})$ were incubated with HLC fractions $(1 \mathrm{mg} / \mathrm{ml})$ and GSH $(1000 \mu \mathrm{M})$ under the same conditions. At predetermined time intervals $(0,5,15,30$, and 60 minutes) ice-cold acetonitrile containing $1 \mathrm{mM} \mathrm{NEM}$ was added to terminate the reactions. All terminated samples were stored at $-80^{\circ} \mathrm{C}$ until the quantitative determination of thioether metabolites by liquid chromatography-tandem mass spectrometry (LC-MS/MS).

Incubation of PPI Thioethers in HLMs and FMOs. The conditions for incubation in HLMs were optimized to be linear with respect to incubation time and protein concentrations. The final incubation mixture contained substrate, HLMs $(0.1 \mathrm{mg} / \mathrm{ml})$, and NADPH $(1 \mathrm{mM})$ in a final volume of $200 \mu \mathrm{l}$ of $100 \mathrm{mM}$ PBS ( $\mathrm{pH}$ 7.4). The mixtures were preincubated for 5 minutes at $37^{\circ} \mathrm{C}$, and each reaction was initiated by adding various concentrations of PPI thioethers $(0.4,2,4$, $8,16,32,48,80$, and $200 \mu \mathrm{M})$. The control HLM was heat inactivated at $45^{\circ} \mathrm{C}$ for 2 minutes, wherein the FMO activity was abolished but not the P450s. After incubation at $37^{\circ} \mathrm{C}$ for 10 minutes, $400 \mu \mathrm{l}$ of acetonitrile containing $0.1 \%$ diethylamine and an internal standard [200 $\mathrm{ng} / \mathrm{ml}(R)$-LAN-d4] was added to terminate the reaction. The mixtures were vortexed for 1 minute and centrifuged at

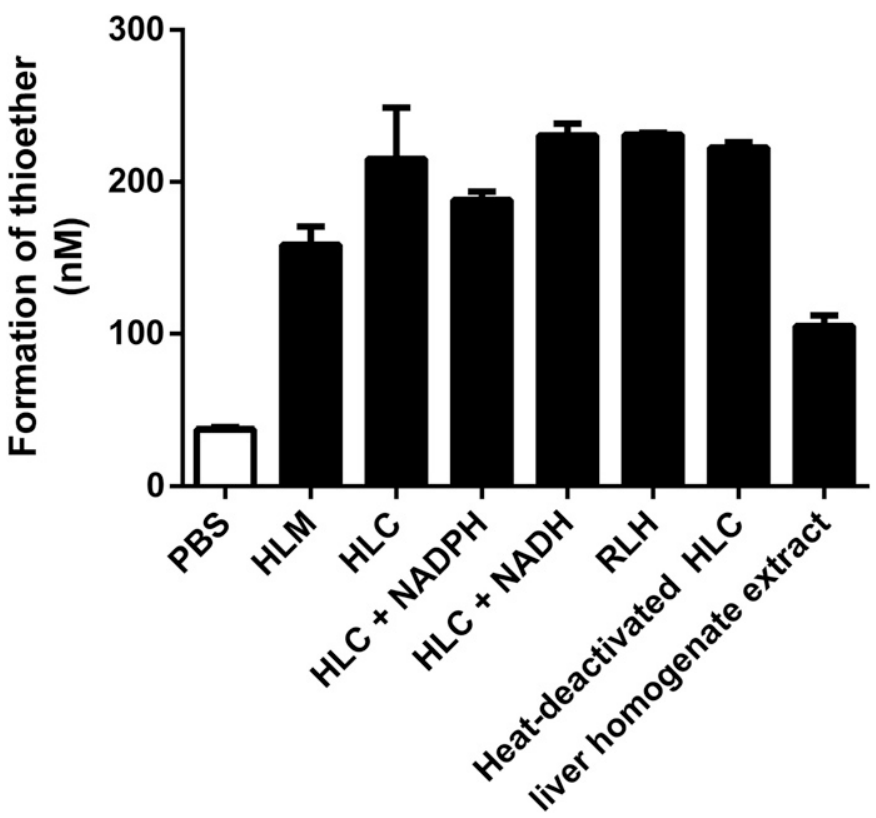

Fig. 2. Amount of formed $\mathrm{RAB}$ thioether after incubation of RAB $(1.5 \mu \mathrm{M})$ in different liver subcellular fractions. Results are expressed as mean \pm S.D. $(n=3)$.

$13,000 \mathrm{~g}$ at $4^{\circ} \mathrm{C}$ for 5 minutes. A $100 \mu \mathrm{l}$ aliquot of the supernatant was diluted 10 -fold with the mobile phase, and then $5 \mu \mathrm{l}$ of the resulting solution was injected into the LC-MS/MS system to determine the concentrations of $(R)$ - and $(S)$-PPIs. The experiments were performed in duplicate. Incubations of PPI thioethers were also conducted in recombinant FMOs. The PPI thioethers were added at a final concentration of $3 \mu \mathrm{M}$ (DMSO $0.1 \%$ ) to a final volume of $100 \mu \mathrm{l}$ of PBS (100 mM, pH 7.4). A stock solution of FMOs (FMO1, FMO3, and FMO5) or HLMs was also added at final concentrations of $1.0 \mathrm{mg} / \mathrm{ml}$. The mixtures were preincubated at $37^{\circ} \mathrm{C}$ for 3 minutes, and then the reactions were initiated by the addition of NADPH at a final protein concentration of $1 \mathrm{mM}$. The mixtures were incubated at $37^{\circ} \mathrm{C}$ for 60 minutes and quenched by the addition of an equal volume of acetonitrile containing $0.1 \%$ diethylamine. All terminated samples were stored at $-80^{\circ} \mathrm{C}$ until the quantitative determination of $(R)$ - and $(S)$-PPIs by chiral LC-MS/MS

Sample Preparation. $(R)$-LAN-d4 $(25 \mu \mathrm{l})$ in methanol $(200 \mathrm{ng} / \mathrm{ml})$ was added as an internal standard to mixtures of $25 \mu 1$ of plasma or incubation samples and $200 \mu \mathrm{l}$ of acetonitrile containing $0.1 \%$ diethylamine. The mixtures were vortexed for 1 minute and centrifuged at $13,000 \mathrm{~g}$ at $4^{\circ} \mathrm{C}$ for 5 minutes. Then, $1 \mu \mathrm{l}$ of the supernatant was injected into the LC-MS/MS system.

Determination of PPI Enantiomers and Thioether Metabolites in Plasma or Incubation Samples by Chiral LC-MS/MS. The high-performance liquid

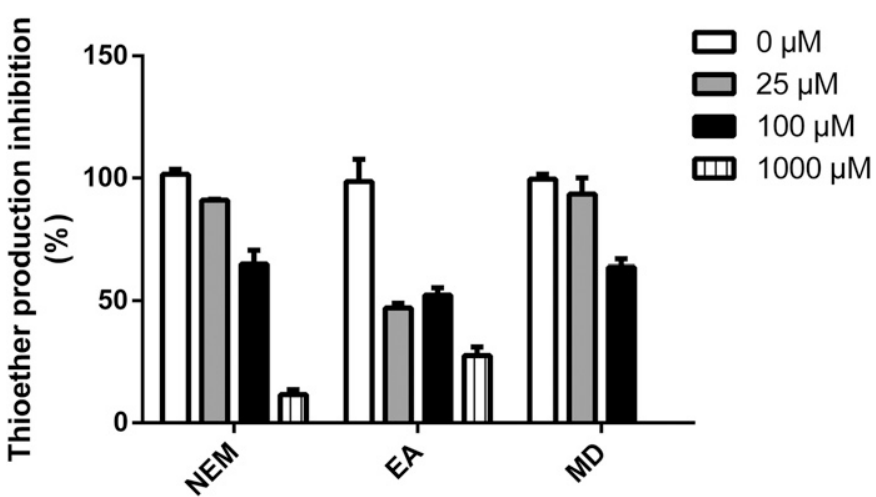

Fig. 3. Amount of formed RAB thioether after incubation of RAB $(1.5 \mu \mathrm{M})$ in HLC fractions treated with different concentrations of NEM, EA, and MD. Results are expressed as mean \pm S.D. $(n=3)$. 

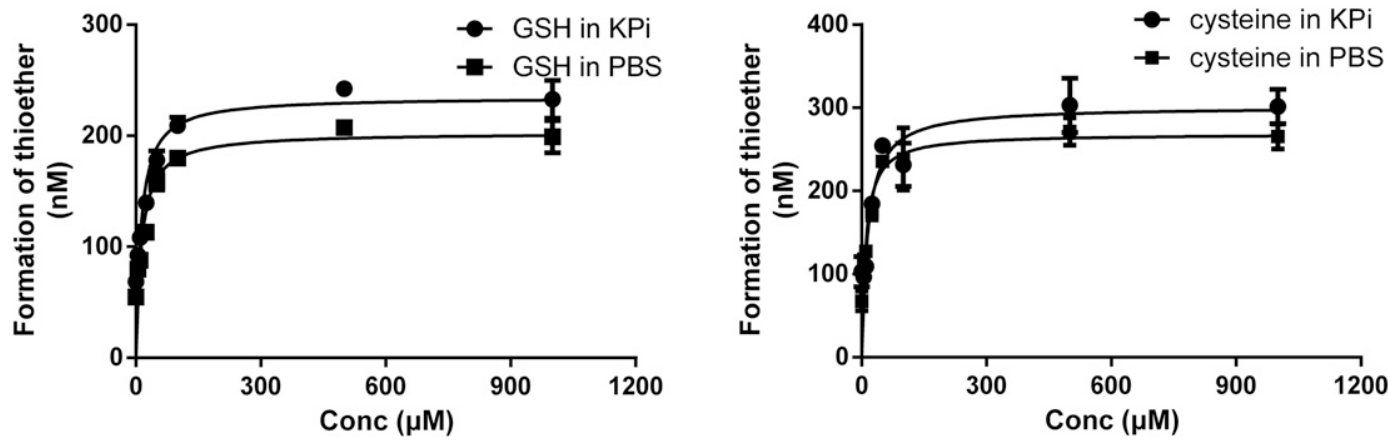

Fig. 4. Amount of formed RAB thioether after incubation of racemic RAB $(1.5 \mu \mathrm{M})$ with different concentrations of GSH and cysteine in PBS and potassium phosphate (KPi) solutions. Results are expressed as mean \pm S.D. $(n=3)$.

chromatography system consisted of an LC-30AD pump equipped with an SIL30AC autosampler (Shimadzu, Kyoto, Japan). Chiral separation of PPI enantiomers was performed on Lux Cellulose- 4 chiral columns $(150 \times 4.6 \mathrm{~mm}, 5 \mu \mathrm{M})$ from Phenomenex (Torrance, $\mathrm{CA}$ ) at $40^{\circ} \mathrm{C}$ with a flow rate of $0.8 \mathrm{ml} / \mathrm{min}$. The separation was carried out using a gradient elution procedure. The mobile phase $\mathrm{A}$ (5 $\mathrm{mM}$ ammonium acetate) and B [acetonitrile/isopropanol $(30: 70, \mathrm{v} / \mathrm{v})]$ ratios linearly changed as follows: $0-0.8$ minutes, $25 \% \mathrm{~B} ; 0.8-2.1$ minutes, $25 \%-43 \%$ B; 2.1-3.6 minutes, $43 \%-55 \%$ B; 3.6-4.6 minutes, $55 \%-95 \%$ B; and 4.64.8 minutes, $95 \% \mathrm{~B}$. Then, the column was equilibrated with the initial mobile phase. When analyzing PAN enantiomers, the gradient elution procedure was slightly modified to obtain better resolution. The total run time was 6.5 minutes.

MS detection was performed using an AB Sciex Triple Quad 6500 System (Applied Biosystems, Concord, ON, Canada) equipped with a TurboIonSpray ion source. Multiple reactions monitoring was used to quantify compounds in the positive ion mode [mass-to-charge ratio $(\mathrm{m} / \mathrm{z}) 330.1 \rightarrow 182.1$ for OME thioether, $\mathrm{m} / \mathrm{z}, 346.1 \rightarrow 198.1$ for OME, $\mathrm{m} / \mathrm{z}, 354.2 \rightarrow 236.2$ for LAN thioether, $\mathrm{m} / \mathrm{z}, 370.2 \rightarrow$ 252.2 for LAN, $\mathrm{m} / \mathrm{z} 368.2 \rightarrow 184.2$ for PAN thioether, $\mathrm{m} / \mathrm{z} 384.2 \rightarrow 200.2$ for PAN, $m / z, 344.2 \rightarrow 226.2$ for RAB thioether, and $m / z \quad 360.2 \rightarrow 242.2$ for RAB].

Sample Preparation and Ultra-Performance Liquid Chromatography/QTOF MS Analysis. The reductive metabolism of PPIs in HLC fractions was significantly inhibited by sulfhydryl-blocking agents (NEM, EA, and MD). Supposedly, certain reductive agents were depleted by these compounds. Acetonitrile $(800 \mu \mathrm{l})$ was added to a $200 \mu \mathrm{l}$ aliquot of the HLC fraction incubations that were treated with NEM, EA, or MD and GSH incubations. These samples were vortex mixed and centrifuged at $14,000 \mathrm{~g}$ for 5 minutes. The supernatant was transferred into a plastic tube, evaporated to dryness under a stream of nitrogen at $40^{\circ} \mathrm{C}$, and then reconstituted in $120 \mu \mathrm{l}$ of water/methanol (98:2, v/v) solution. A $7 \mu \mathrm{l}$ aliquot of the reconstituted solution was injected into the ultra-performance liquid chromatography Q-TOF Synapt G2 system (Waters, Milford, MA) for analysis. The rat liver S9 incubations were prepared as described previously, except that the reconstitution reagent was replaced with acetonitrile containing $0.1 \%$ diethylamine as a stabilizer.
The analytes in the GSH incubations and inhibitor-treated HLC fraction incubations were separated with a mobile phase consisting of deionized water containing $0.1 \%$ formic acid as the aqueous phase and methanol as the organic phase by using an Acquity HSS $\mathrm{T}_{3}$ column $(100 \times 2.1 \mathrm{~mm}, 1.8 \mu \mathrm{M})$ equilibrated at $40^{\circ} \mathrm{C}$. The column was eluted with a linear gradient of $2 \%-50 \% \mathrm{~B}$ over an initial time to 6.0 minutes, $50 \%-70 \%$ B over $2.0-7.0$ minutes, and $70 \%-99 \%$ B over 7.0-11.0 minutes, and then held at $99 \%$ B for 1.0 minute, returned to $1 \% \mathrm{~B}$ for 0.5 minutes, and held for 1.5 minutes at an eluent flow rate of $0.40 \mathrm{ml} / \mathrm{min}$. The chromatographic separation of analyte in rat liver S9 incubations was conducted using Lux Cellulose- 4 chiral columns $(150 \times 4.6 \mathrm{~mm}, 5 \mu \mathrm{M})$ from Phenomenex at $40^{\circ} \mathrm{C}$ with a flow rate of $0.8 \mathrm{ml} / \mathrm{min}$. The mobile phase was a mixture of $5 \mathrm{mM}$ ammonium acetate (A) and acetonitrile/isopropanol (30: 70, v/v) (B). The gradient was started from $30 \% \mathrm{~B}$, maintained for 3 minutes, and then increased linearly to $50 \% \mathrm{~B}$ over the next 11 minutes. Afterward, the gradient was rapidly increased to $99 \% \mathrm{~B}$, maintained for 1 minute, reduced to $30 \% \mathrm{~B}$ for 1 minute, and then finally maintained at $30 \%$ B for 2.5 minutes to equilibrate the column. For Q-TOF MS analysis, the optimal conditions were as follows: capillary voltage of $3000 \mathrm{~V}$, desolvation temperature of $450^{\circ} \mathrm{C}$, sample cone voltage of $35 \mathrm{~V}$, source temperature of $120^{\circ} \mathrm{C}$, cone gas flow of $50 \mathrm{l} / \mathrm{h}$, and desolvation gas flow of $800 \mathrm{l} / \mathrm{h}$. The mass spectrometric $\mathrm{MS}^{\mathrm{E}}$ data were acquired in the positive ion mode from 50 to $1200 \mathrm{Da}$ with a 0.15 second scan time at a constant collision energy setting of $2 \mathrm{~V}$ during the low-energy MS mode (function 1) for precursor ion data, and then dynamically from 15 to $40 \mathrm{~V}$ collision energy during the high-energy $\mathrm{MS}^{\mathrm{E}}$ mode (function 2) for optimal fragmentation data. Mass was corrected during acquisition using an external reference (LockSpray) consisting of a $40 \mathrm{ng} / \mathrm{ml}$ solution of leucine enkephalin infused at a flow rate of $10 \mu \mathrm{l} / \mathrm{min}$ via a LockSpray interface, generating a reference ion for positive ion mode $\left([\mathrm{M}+\mathrm{H}]^{+}=\right.$ 556.2771) to ensure accuracy during the MS analysis.

Data Analysis. WinNonlin (version 6.1; Pharsight Corp., Cary, NC) was used to calculate the pharmacokinetic parameters in a noncompartmental model. GraphPad Prism (version 5.0; GraphPad Software Inc., San Diego, CA) was used to generate the $K_{\mathrm{m}}$ and $V_{\max }$ values. All data are expressed as mean \pm S.D.
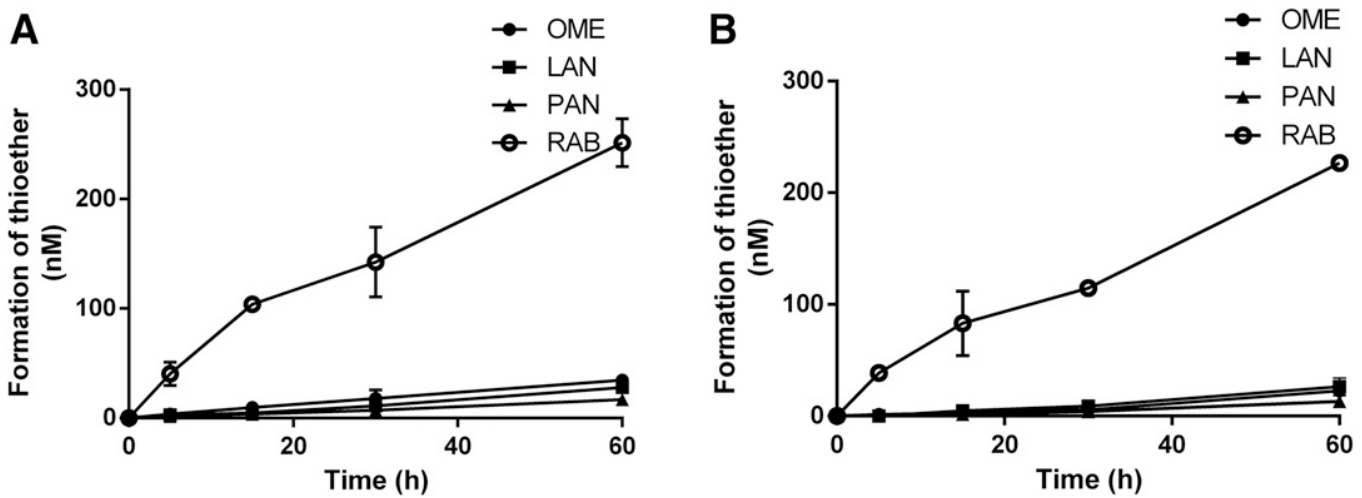

Fig. 5. Formation of thioether metabolites after incubation of four racemic PPIs $(1.5 \mu \mathrm{M})$ in GSH solution $(1000 \mu \mathrm{M})(\mathrm{A})$ and HLC fractions (B). Results are expressed as mean \pm S.D. $(n=3)$. 
TABLE 3

Formation rate (nmol/l per minute) of PPI thioethers in GSH and HLC fractions Results are expressed as mean \pm S.D. $(\mathrm{n}=3)$.

\begin{tabular}{lcccc}
\hline $\begin{array}{c}\text { Incubation } \\
\text { System }\end{array}$ & PAN & LAN & OME & RAB \\
\hline GSH & $0.281 \pm 0.025$ & $0.460 \pm 0.094$ & $0.573 \pm 0.028$ & $4.33 \pm 0.37$ \\
HLC fractions & $0.218 \pm 0.019$ & $0.354 \pm 0.012$ & $0.377 \pm 0.016$ & $3.78 \pm 0.02$ \\
\hline
\end{tabular}

\section{Results}

Stereoselective Oxidation of RAB Thioether in SD Rats. To test whether RAB thioether could be oxidized to parent drug, rats were administered intravenously with $1 \mathrm{mg} / \mathrm{kg} \mathrm{RAB}$ thioether. The average concentration-time profiles are shown in Fig. 1. $(R)$-RAB and $(S)$-RAB were observed in rat plasma from the first blood sampling time. RAB thioether and $\mathrm{RAB}$ enantiomers were all eliminated rapidly from plasma. The biotransformation of RAB thioether to $(R)$ - and $(S)$-enantiomers was stereoselective. The plasma concentration of $(R)-\mathrm{RAB}$ was significantly higher than that of $(S)$-RAB.

Stereoselective Oxidation of PPI Thioethers in HLMs and FMOs. Thioether metabolites of OME, LAN, PAN, and RAB were incubated with HLMs, and the concentrations of the corresponding $(R)$ - and $(S)$-enantiomers were determined by LC-MS/MS. Nonlinear regression analysis was used to calculate the kinetic parameters $\left(K_{\mathrm{m}}\right.$ and $\left.V_{\max }\right)$, as shown in Table 1 . The oxidative metabolisms of PPI thioethers to form sulfoxides were all stereoselective. In addition to PAN thioether, three other PPI thioethers were preferentially metabolized to their $(R)$-enantiomers. $(R)$-PPIs and $(S)$-PPIs were also detected in thioetherfortified incubated samples with FMO1, FMO3, and FMO5; however, the yields were significantly lower than those in the HLM incubations (Supplemental Fig. 1), and the formation in FMO-deactivated liver microsomes (by heating) showed no difference from that in the untreated liver microsomes. Therefore, P450s were considered as the main enzymes involved in the oxidation of thioethers to PPIs.

Formation Region of RAB Thioether In Vivo. The AUC data obtained for the parent drug and $\mathrm{RAB}$ thioether after administration of $(R)$ - RAB to rats via different routes are given in Table 2. Following oral administration, the concentrations of RAB and RAB thioether in portal vein were both higher than those in systemic circulation; however, the molar $\mathrm{AUC}_{0-t}$ ratios of thioether to $\mathrm{RAB}$ in the portal vein were significantly lower than those in systemic circulation ( $11.8 \%$ vs. $30.6 \%$ ). The molar $\mathrm{AUC}_{0-t}$ ratios were $15.5 \%$ and $28.6 \%$ in rats administered via intravenous and portal vein routes, respectively. These data indicated that RAB thioether was mainly formed in the liver instead of the intestine.

Metabolism of $(R)-R A B$ in Liver Subcellular Fractions. To elucidate the formation pathway of RAB thioether, $(R)-\mathrm{RAB}$ was incubated with liver subcellular fractions and PBS. The amount of produced RAB thioether is shown in Fig. 2. After 1-hour incubation, approximately $13.3 \%$ of RAB was transformed into thioether in HLC fractions, a similar amount of thioether was formed in rat liver homogenate, and a slightly lower amount was found in HLMs. However, only $2.35 \%$ was found in PBS. The formation of thioether was not increased with the addition of NADPH or NADH to HLC fraction incubations. Heat-deactivated HLC fractions (in which HLC fractions were heated for 10 minutes in a $100^{\circ} \mathrm{C}$ water bath) had the same reducing capacity. These data indicated that the reductive metabolism of RAB in liver subcellular fractions was not mediated by liver reductases.

Further studies were conducted to confirm the dominant components that were involved in the reductive metabolism of PPIs. As shown in Fig. 3, concentration-dependent inhibition of reductive metabolism was
A

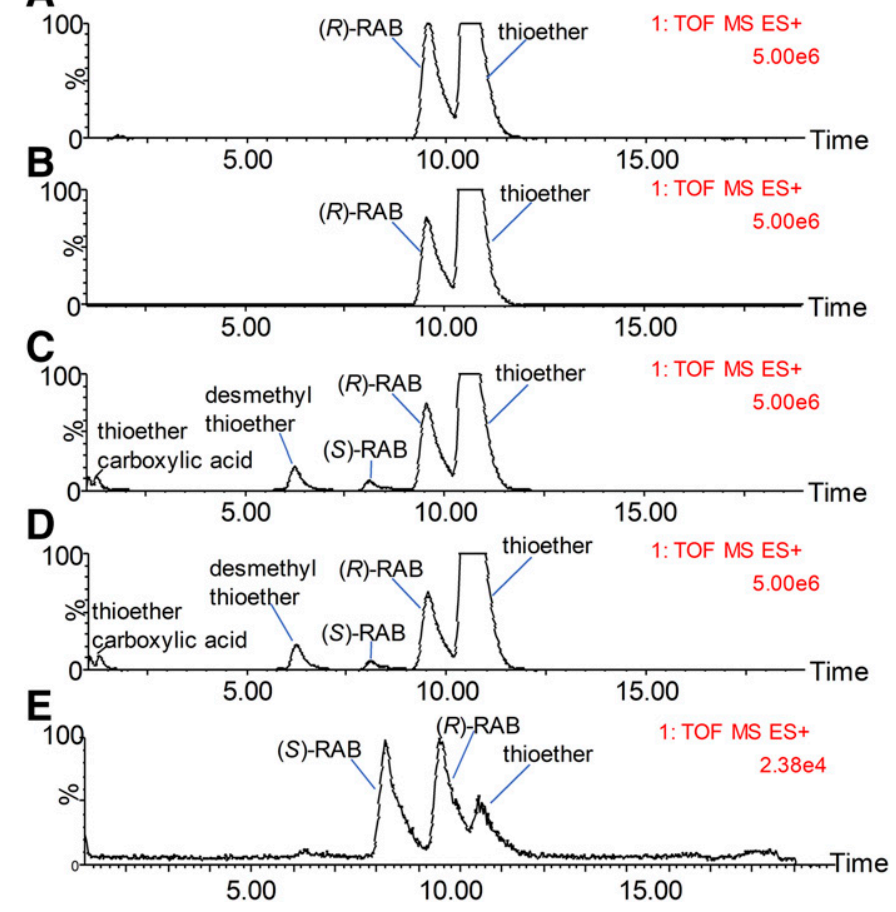

Fig. 6. MS detection of the main metabolic profiles of $(R)$-RAB in rat liver $\mathrm{S} 9$ in the absence of both NADPH and GSH (A), in the presence of GSH but not NADPH (B), in the presence of GSH but not NADPH (C), and in the presence of both NADPH and GSH (D). A chromatogram of the mixture solution of racemic RAB $(50 \mathrm{nM})$ and RAB thioether $(10 \mathrm{nM})$ iss provided in $(\mathrm{E})$.

observed when sulfhydryl-blocking agents (NEM, EA, and MD) were added to the HLC fraction incubations. The amount of produced thioether was reduced to $64.9 \%, 52.3 \%$, and $63.7 \%$ at $100 \mu \mathrm{M}$ of NEM, $\mathrm{EA}$, and MD, respectively. With further increasing concentrations of the three inhibitors, the reductive metabolism was nearly completely inhibited.

Reductive Reaction of RAB in GSH and Cysteine Solutions. $\mathrm{RAB}$ thioether was detected after the incubation of RAB in GSH and cysteine solutions for 1 hour. It was formed in a GSH or cysteine concentration-dependent manner (Fig. 4) when the concentration of GSH or cysteine was less than 10-fold of the substrate. However, the formation did not continue to increase when the GSH or cysteine concentration was increased to normal intracellular levels $(1 \mathrm{mM})$. In addition, there was no significant difference in the rate of reduction of RAB in PBS and potassium phosphate solutions containing GSH or cysteine.

The formation rates of thioether metabolites of the four PPIs were measured in GSH solution and HLC fractions. As shown in Fig. 5, the formation of all four thioethers in both incubation systems was time dependent within 60 minutes, and the formation rate of the four PPI thioethers differed. In both incubation systems, the formation rate of $\mathrm{RAB}$ was the fastest. The rank order of reduction rates in GSH was $\mathrm{RAB}>\mathrm{OME}>\mathrm{LAN}>\mathrm{PAN}$ (Table 3 ). In the incubation of RAB with GSH, the two GSH conjugates identified as benzimidazole-GSH and [RAB- $\left.\mathrm{H}_{2} \mathrm{O}\right]-\mathrm{GSH}$ were observed in a GSH concentration-dependent manner (Supplemental Fig. 2).

Reductive Metabolism and Chiral Inversion in Rat Liver S9 Fractions. The main metabolic profiles of $(R)-\mathrm{RAB}$ in rat liver $\mathrm{S} 9$ incubations are shown in Fig. 6. $(R)-\mathrm{RAB},(S)-\mathrm{RAB}$, and RAB thioether were confirmed using reference standards. The results showed that $(R)$-RAB can be converted to thioether regardless of whether GSH was added in rat liver $\mathrm{S} 9$. The formation rate of $\mathrm{RAB}$ thioether is similar to 
A

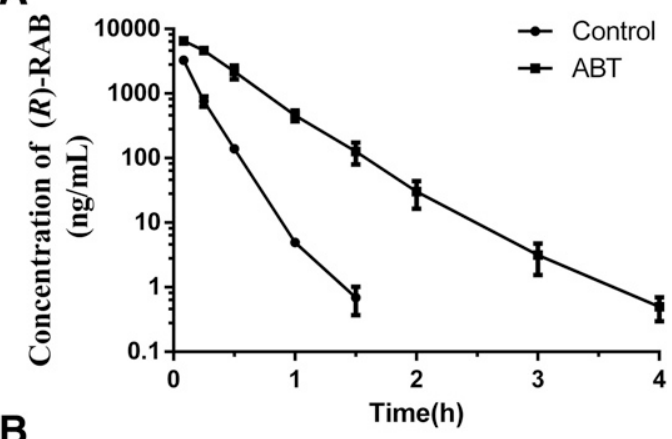

B
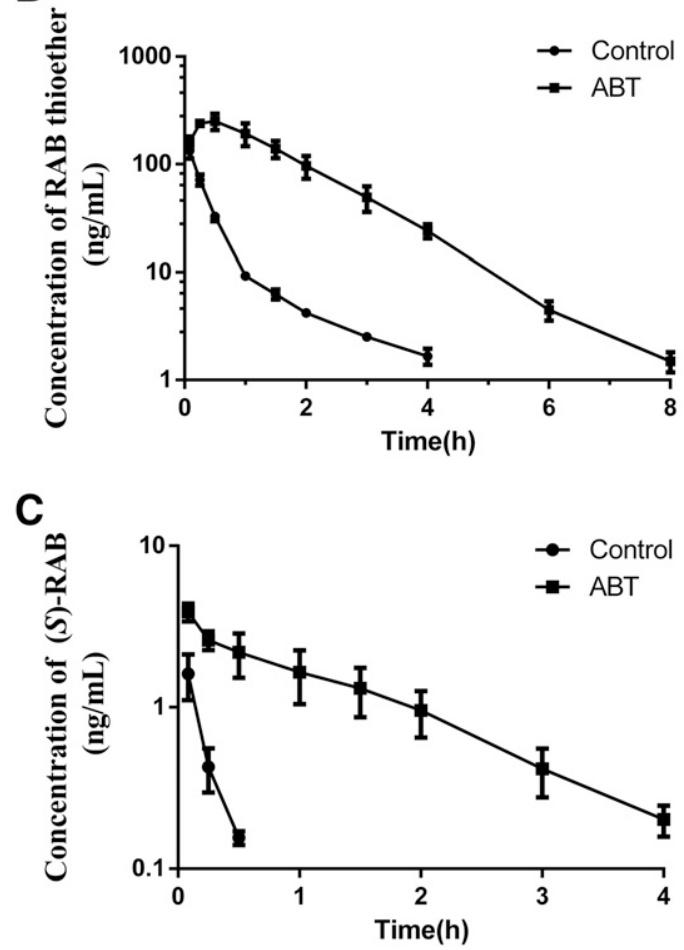

Fig. 7. Mean plasma concentration-time profiles of $(R)$-RAB (A), RAB thioether (B), and (S)-RAB (C) of ABT-treated (100 mg/kg) rats and control rats after intravenous doses of $(R)-\mathrm{RAB}(3 \mathrm{mg} / \mathrm{kg})$. Data are presented as mean \pm S.D. $(n=4)$

that of other liver subcellular fractions. In the incubation of $(R)$-RAB with NADPH-supplemented rat S9, chiral inversion metabolite $(S)$-RAB and other thioether-related metabolites such as desmethyl thioether and thioether carboxylic acid were observed.

Effect of Inhibition of P450 Enzymes on Reductive Metabolism and Chiral Inversion. The average concentration-time profiles of $(R)-\mathrm{RAB},(S)-\mathrm{RAB}$, and $\mathrm{RAB}$ thioether after intravenous administration of $(R)$-RAB are shown in Fig. 7, and the noncompartmental pharmacokinetic parameters of $(R)-\mathrm{RAB},(S)-\mathrm{RAB}$, and $\mathrm{RAB}$ thioether are given in Table 4. In the ABT group, the $\mathrm{AUC}$ of $(R)-\mathrm{RAB}$ was 4.6 times higher than that of the control group, and the AUCs of the reductive metabolite and chiral inversion product in the ABT group were 12.0 and 14.7 higher than those of the control group, respectively. The $\mathrm{AUC}_{0-t}$ ratio of $\mathrm{RAB}$ thioether to $\mathrm{RAB}$ was $22.3 \%$; however, only $8.6 \%$ was found in the control group.

\section{Discussion}

PPIs show varied extent of chiral inversion in vivo. Under most circumstances, the chiral inversion degree of PPIs is small or even
TABLE 4

Pharmacokinetic parameters of $(R)-\mathrm{RAB},(S)$ - $\mathrm{RAB}$, and RAB thioether obtained after intravenous injection of $(R)-\mathrm{RAB}(3 \mathrm{mg} / \mathrm{kg})$ in ABT-treated and control rats

Results are expressed as mean $\pm S . D .(\mathrm{n}=4)$. The em dashes indicate no value because of the lack of time points to calculate.

\begin{tabular}{ccccc}
\hline Component & $\mathrm{AUC}_{0-t}$ & $\mathrm{AUC}_{0-\infty}$ & $t_{1 / 2}$ & MRT \\
\hline & $n g \cdot h / m l$ & $n g \cdot h / m l$ & $h$ & $h$ \\
$(R)-\mathrm{RAB}$ & & & & \\
ABT & $2858 \pm 325$ & $2891 \pm 354$ & $0.273 \pm 0.052$ & $0.360 \pm 0.008$ \\
Control & $621 \pm 72$ & $621 \pm 73$ & $0.122 \pm 0.009$ & $0.165 \pm 0.007$ \\
Thioether & & & & \\
ABT & $609 \pm 146$ & $610 \pm 147$ & $1.02 \pm 0.08$ & $1.55 \pm 0.13$ \\
Control & $50.8 \pm 2.8$ & $50.9 \pm 2.8$ & $1.01 \pm 0.11$ & $0.656 \pm 0.043$ \\
$(S)$-RAB & & - & $0.913 \pm 0.070$ & $1.19 \pm 0.08$ \\
ABT & $4.57 \pm 1.10$ & - & - & $0.159 \pm 0.015$ \\
Control & $0.31 \pm 0.08$ & - & &
\end{tabular}

$\mathrm{AUC}_{0-\infty}$, AUC from time 0 to infinity; MRT, mean residence time; $t_{1 / 2}$, half-life.

negligible (Andersson et al., 2001; Xie et al., 2004; Gao et al., 2013). However, obvious chiral inversion caught our attention in the phase I clinical trial of $(R)$-anaprazole, a novel PPI with chemical properties and metabolic profiles similar to RAB (Fig. 8).

Thioether drugs such as cimetidine, sulindac thioether, and flosequinan thioether can all be oxidized to sulfoxide form by flavin monooxygenase or P450 enzymes (Mitchell et al., 1982; Kashiyama et al., 1997; Hamman et al., 2000). In our research, all of the four PPI thioethers were stereoselectively oxidized to $(R)$-PPIs and $(S)$-PPIs after incubation in HLMs. After the intravenous administration of RAB thioether, $(R)-\mathrm{RAB}$ and $(S)$-RAB were both detected in rat plasma. Based on the in vivo and in vitro results, thioether metabolites of PPIs may be the intermediate of chiral inversion of PPIs.

Xenobiotics containing a sulfoxide moiety such as sulindac and fenthion sulfoxide can be reduced by several reductases, including aldehyde oxidase and methionine sulfoxide reductase, in the liver (Tatsumi et al., 1982; Tarrago et al., 2018). The sulfoxide reduction of flosequinan is mediated by intestinal bacteria in the small intestine (Kashiyama et al., 1994). Reductive metabolism to form thioether is also a common metabolic pathway of PPIs; however, the main organ and reductases of the reductive metabolism have not been reported (Fuhr and Jetter, 2002; Pu et al., 2018). By injection via the portal vein, drugs can enter into systemic circulation without undergoing first-pass metabolism in the intestine (Kunta et al., 2004; Shin et al., 2014). Rats were administered $(R)-\mathrm{RAB}$ via different routes, and the molar $\mathrm{AUC}_{0-t}$ ratios of thioether to $\mathrm{RAB}$ in the portal vein were significantly lower than those in systemic circulation after oral administration. The $\mathrm{AUC}_{0-t}$ ratio in rats administered via portal vein was also higher than that of the intravenous group, respectively. These data indicated that RAB thioether was mainly formed in the liver instead of the intestine.

$\mathrm{RAB}$ was chemically transformed into thioether spontaneously in PBS, while a higher reaction rate in liver subcellular fractions was observed, indicating that reductases or reducing agents were involved in the reductive pathway of PPIs. However, NADPH and NADH were not essential in the reductive process, and even the heat-deactivated HLC fractions also possessed the same reductive capacity as normal HLC fractions. The reduction rate in the extract of freshly prepared rat liver homogenates by acetonitrile was higher than that in PBS. These results proved that $\mathrm{RAB}$ was converted into $\mathrm{RAB}$ thioether mainly via nonenzymatic metabolism, and some biologic reducing agents might be involved in the reductive metabolism of PPIs. Sulfhydryl-blocking agents (NEM, MD, and EA) inhibited the reductive metabolism of RAB in liver subcellular fractions in a concentration-dependent manner. Moreover, $\alpha$ - $\beta$-unsaturated ketones/amides were contained in these 


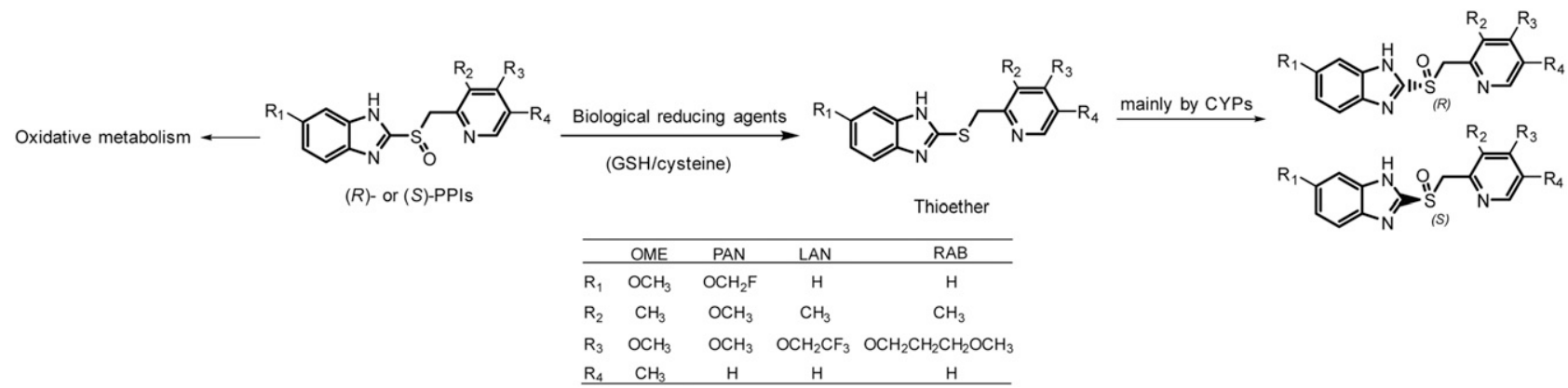

Fig. 8. Proposed mechanism of chiral inversion of PPIs and structures of commonly used PPIs. The general structures of PPIs are given. R represents different substituent groups (numbers represent different substituents).

compounds. Michael addition products between these compounds and GSH were detected in inhibitor-treated HLC fractions (Supplemental Fig. 3), suggesting that the inhibition of reduction reaction may be caused by the depletion of the thiols in liver subcellular fractions.

In addition to GSH, other thiol compounds such as cysteine and dithiothreitol accelerated the reduction rate of PPIs in PBS to the same degree as HLC fractions. Thiol depletors such as iodoacetamide also inhibited the reductive metabolism of PPIs in HLC fractions (data not shown). These results revealed that the reductive metabolism of PPIs in vivo and in subcellular fractions was mediated by endogenous biologic reducing agents such as GSH and cysteine.

A marked difference exists between the in vivo concentrations of GSH and PPIs. The $C_{\max }$ value of PPIs in human plasma after oral administration of OME (20 or $40 \mathrm{mg}$ ), LAN (30 mg), PAN (40 mg), and RAB (20 mg) was only approximately $0.534-9.28 \mu \mathrm{M}$ (Huber et al., 1995; Andersson et al., 2001; Miura et al., 2005; Dash et al., 2018). However, the physiologic concentrations of GSH in hepatocytes reached $10 \mathrm{mM}$, and the levels of other endogenous thiol compounds were also significantly higher than those of PPIs (Lörincz and Szarka, 2017; Zarka and Bridge, 2017). The present study showed that when the concentration of GSH increased to more than 10 times that of PPIs, the formation rate of thioethers no longer increased. Therefore, as long as PPIs enter the liver, they are transformed into thioethers at a constant and maximum rate, and the reductive metabolism of PPIs is not influenced by the physiologic fluctuation of GSH concentrations. Although moderate levels of GSH were observed in whole blood (Zarka and Bridge, 2017), PPIs were not reduced to thioether in fresh rat and human whole blood, probably because of the high degree of binding with plasma protein.

The degree of chiral inversion was directly affected by the degree of reductive metabolism of PPIs. The formation rate of RAB thioether in GSH solutions and HLC fractions was significantly higher than that of other PPIs. The plasma exposure of RAB thioether in humans accounted for approximately 50\% of the parent drugs (Shirai et al., 2001; Miura et al., 2006); however, the plasma concentrations of OME thioether and LAN thioether were low, which could explain why the chiral inversion of OME and LAN in humans was negligible. Meanwhile, a positive correlation was found between the natural bond orbital charges (Reed et al., 1985, 1988) of sulfur atoms in these compounds and the reaction rate (Cheshmedzhieva et al., 2009) in GSH (Supplemental Table 1). The change of substituents affected the oxidation-reduction properties of PPIs and their elimination pathways in vivo.

PPIs were eliminated from the circulation by extensive metabolism (Horn, 2004). Oxidative metabolism was the main clearance pathway for OME, LAN, and PAN, whereas reductive metabolism was the minor pathway for them (Li et al., 1992; Rezk et al., 2006). However, a new class of PPIs, including RAB and ilaprazole, was mainly metabolized via reduction to thioether (Dash et al., 2018; Pu et al., 2018). A mass balance study showed that after oral dosing of $\left[{ }^{14} \mathrm{C}\right] \mathrm{RAB}$, the main radioactive components were thioether carboxylic acid and mercapturic acid conjugate metabolites in urine and thioether carboxylic acid in the feces, suggesting that oxidative metabolism was also needed for the final clearance of RAB (Setoyama et al., 2006). The plasma exposure of RAB thioether in humans was influenced by the activity of oxidative enzymes (Uno et al., 2006). In poor metabolizers of CYP2C19, the mean AUC values of RAB thioether were found to be 2.68 times higher than those in extensive metabolizers (Miura et al., 2005). Therefore, we evaluated the influence of $\mathrm{P} 450$ activities on the degree of reductive metabolism and chiral inversion of PPIs. After the inhibition of P450s by ABT in SD rats, the AUCs of RAB thioether and chiral inversion product were both markedly increased. On the one hand, further metabolism of thioether to form thioether carboxylic acid was inhibited and the direct oxidative metabolism of $(R)$-RAB was also blocked. Therefore, more RAB were reduced to thioether as compensation. Chemical inhibition or activity reduction of oxidative enzymes increased the plasma exposure of RAB thioether and then the degree of chiral inversion.

The mechanism of chiral inversion of PPIs in vivo was elucidated in our study. PPIs were metabolized to thioethers in the liver by biologic reducing agents such as GSH and cysteine, and then thioether metabolites were stereoselectively oxidized to $(R)$ - and $(S)$-PPIs to cause chiral inversion (Fig. 8). The study is instructive for the development of single enantiomer PPIs and avoiding drug-drug interactions in clinical use due to increased plasma exposure of thioether metabolites.

\section{Acknowledgments}

We greatly appreciate Aosaikang Pharmaceutical (Jiangsu, China) for providing $(R)$ - and $(S)$-rabeprazole.

\section{Authorship Contributions \\ Participated in research design: Tang, X. Chen. Conducted experiments: Tang, Z. Chen, D. Xiaojian. \\ Contributed new reagents or analytic tools: Tang, Zhu, Zhong, X. Chen. \\ Performed data analysis: Tang, Z. Chen, Zhu, X. Chen. \\ Wrote or contributed to the writing of the manuscript: Tang, X. Chen.}

\section{References}

Agranat I, Caner H, and Caldwell J (2002) Putting chirality to work: the strategy of chiral switches. Nat Rev Drug Discov 1:753-768.

Andersson T, Hassan-Alin M, Hasselgren G, Röhss K, and Weidolf L (2001) Pharmacokinetic studies with esomeprazole, the (S)-isomer of omeprazole. Clin Pharmacokinet 40:411-426.

Bourland JA, Martin DK, and Mayersohn M (1998) In vitro transesterification of cocaethylene (ethylcocaine) in the presence of ethanol. esterase-mediated ethyl ester exchange esterasemediated ethyl ester exchange. Drug Metab Dispos 26:203-206.

Cheshmedzhieva D, Ilieva S, Hadjieva B, Trayanova T, and Galabov B (2009) Reactivity of acetanilides in the alkaline hydrolysis reaction: theory vs. experiment. Mol Phys 107:1187-1192. Dash RP, Rais R, and Srinivas NR (2018) Stereoselective and nonstereoselective pharmacokinetics of rabeprazole-an overview. Xenobiotica 48:422-432. 
Esterbauer H, Zollner H, and Lang J (1985) Metabolism of the lipid peroxidation product 4-hydroxynonenal by isolated hepatocytes and by liver cytosolic fractions. Biochem $J \mathbf{2 2 8}$ : 363-373.

Freedberg DE, Kim LS, and Yang YX (2017) The risks and benefits of long-term use of proton pump inhibitors: expert review and best practice advice from the American Gastroenterological Association. Gastroenterology 152:706-715.

Fuhr U and Jetter A (2002) Rabeprazole: pharmacokinetics and pharmacokinetic drug interactions. Pharmazie 57:595-601.

Gao YH, Xu JX, Su ZX, Song L, and Lou HX (2013) The chiral bioconversion and preclinical pharmacokinetic analysis of $(R)-(+)$-rabeprazole in beagle dogs by HPLC and HPLC-MS/MS Biomed Chromatogr 27:1380-1386.

Hamman MA, Haehner-Daniels BD, Wrighton SA, Rettie AE, and Hall SD (2000) Stereoselective sulfoxidation of sulindac sulfide by flavin-containing monooxygenases. Comparison of human liver and kidney microsomes and mammalian enzymes. Biochem Pharmaco 60:7-17.

Horn J (2004) Review article: relationship between the metabolism and efficacy of proton pump inhibitors-focus on rabeprazole. Aliment Pharmacol Ther 20 (Suppl 6):11-19.

Huber R, Kohl B, Sachs G, Senn-Bilfinger J, Simon WA, and Sturm E (1995) Review article: the continuing development of proton pump inhibitors with particular reference to pantoprazole. Aliment Pharmacol Ther 9:363-378.

Ikuta H, Kawase A, and Iwaki M (2017) Stereoselective pharmacokinetics and chiral inversion of ibuprofen in adjuvant-induced arthritic rats. Drug Metab Dispos 45:316-324.

Ishizaki T and Horai Y (1999) Review article: cytochrome P450 and the metabolism of proton pump inhibitors - emphasis on rabeprazole. Aliment Pharmacol Ther 13 (Suppl 3):27-36.

Kashiyama E, Yokoi T, Odomi M, Funae Y, Inoue K, and Kamataki T (1997) Cytochrome P450 responsible for the stereoselective $S$-oxidation of flosequinan in hepatic microsomes from rats and humans. Drug Metab Dispos 25:716-724.

Kashiyama E, Yokoi T, Todaka T, Odomi M, and Kamataki T (1994) Chiral inversion of drug: role of intestinal bacteria in the stereoselective sulphoxide reduction of flosequinan. Biochem Pharmacol 48:237-243.

Kunta JR, Lee SH, Perry BA, Lee YH, and Sinko PJ (2004) Differentiation of gut and hepatic firstpass loss of verapamil in intestinal and vascular access-ported (IVAP) rabbits. Drug Metab Dispos 32:1293-1298.

Landes BD, Miscoria G, and Flouvat B (1992) Determination of lansoprazole and its metabolites in plasma by high-performance liquid chromatography using a loop column. J Chromatogr A 577: $117-122$

Lörincz T and Szarka A (2017) The determination of hepatic glutathione at tissue and subcellular level. J Pharmacol Toxicol Methods 88:32-39.

Marom H, Biedermann PU, and Agranat I (2007) Pyramidal inversion mechanism of simple chiral and achiral sulfoxides: a theoretical study. Chirality 19:559-569.

Mitchell SC, Idle JR, and Smith RL (1982) The metabolism of $\left[{ }^{14} \mathrm{C}\right]$ cimetidine in man. Xenobiotica 12:283-292.

Miura M, Tada H, Satoh S, Habuchi T, and Suzuki T (2006) Determination of rabeprazole enantiomers and their metabolites by high-performance liquid chromatography with solid-phase extraction. J Pharm Biomed Anal 41:565-570.

Miura M, Tada H, Yasui-Furukori N, Uno T, Sugawara K, Tateishi T, and Suzuki T (2005) Enantioselective disposition of lansoprazole in relation to CYP2C19 genotypes in the presence of fluvoxamine. Br J Clin Pharmacol 60:61-68.

Pu J, Wang F, Tang W, and Zhu M (2018) Biotransformation of ilaprazole in human liver microsomes and human: role of CYP3A4 in ilaprazole clearance and drug-drug interaction. Drug Metab Dispos 46:1453-1461.

Rayner DR, Gordon AJ, and Mislow K (1968) Thermal racemization of diaryl alkyl aryl and dialkyl sulfoxides by pyramidal inversion. J Am Chem Soc 90:4854-4860.
Reed AE, Curtiss LA, and Weinhold F (1988) Intermolecular interactions from a natural bond orbital, donor-acceptor viewpoint. Chem Rev 88:899-926.

Reed AE, Weinstock RB, and Weinhold F (1985) Natural-population analysis. J Chem Phys 83: 735-746.

Reist M, Carrupt PA, Francotte E, and Testa B (1998) Chiral inversion and hydrolysis of thalidomide: mechanisms and catalysis by bases and serum albumin, and chiral stability of teratogenic metabolites. Chem Res Toxicol 11:1521-1528.

Rezk NL, Brown KC, and Kashuba AD (2006) A simple and sensitive bioanalytical assay for simultaneous determination of omeprazole and its three major metabolites in human blood plasma using RP-HPLC after a simple liquid-liquid extraction procedure. J Chromatogr B Analy Technol Biomed Life Sci 844:314-321.

Setoyama T, Drijfhout WJ, van de Merbel NC, Humphries TJ, and Hasegawa J (2006) Mass balance study of $\left[{ }^{14} \mathrm{C}\right]$ rabeprazole following oral administration in healthy subjects. Int $J$ Clin Pharmacol Ther 44:557-565.

Shin BS, Yoo SD, Kim TH, Bulitta JB, Landersdorfer CB, Shin JC, Choi JH, Weon KY, Joo SH, and Shin S (2014) Quantitative determination of absorption and first-pass metabolism of apicidin, a potent histone deacetylase inhibitor. Drug Metab Dispos 42:974-982.

Shin JM and Kim N (2013) Pharmacokinetics and pharmacodynamics of the proton pump in hibitors. J Neurogastroenterol Motil 19:25-35.

Shirai N, Furuta T, Moriyama Y, Okochi H, Kobayashi K, Takashima M, Xiao F, Kosuge K, Nakagawa K, Hanai H, et al. (2001) Effects of CYP2C19 genotypic differences in the metabolism of omeprazole and rabeprazole on intragastric $\mathrm{pH}$. Aliment Pharmacol Ther 15: 1929-1937.

Tarrago L, Oheix E, Péterfi Z, and Gladyshev VN (2018) Monitoring of methionine sulfoxide content and methionine sulfoxide reductase activity. Methods Mol Biol 1661:285-299.

Tatsumi K, Kitamura S, and Yamada H (1982) Involvement of liver aldehyde oxidase in sulfoxide reduction. Chem Pharm Bull (Tokyo) 30:4585-4588.

Tomaszewski J and Rumore MM (1994) Stereoisomeric drugs: FDA's policy statement and the impact on drug development. Drug Dev Ind Pharm 20:119-139.

Uno T, Shimizu M, Yasui-Furukori N, Sugawara K, and Tateishi T (2006) Different effects of fluvoxamine on rabeprazole pharmacokinetics in relation to CYP2C19 genotype status. Br J Clin Pharmacol 61:309-314.

Uno T, Yasui-Furukori N, Shimizu M, Sugawara K, and Tateishi T (2005) Determination of rabeprazole and its active metabolite, rabeprazole thioether in human plasma by columnswitching high-performance liquid chromatography and its application to pharmacokinetic study. J Chromatogr B Analyt Technol Biomed Life Sci 824:238-243.

Williams RC, Riley CM, Sigvardson KW, Fortunak J, Ma P, Nicolas EC, Unger SE, Krahn DF, and Bremner SL (1998) Pharmaceutical development and specification of stereoisomers. $J$ Pharm Biomed Anal 17:917-924.

Xie ZY, Yang BH, Zhang YF, and Zhong DF (2004) Studies on chiral inversion of dextropantoprazole in human. Үао Хие Хие Вао 39:370-373.

Zarka MH and Bridge WJ (2017) Oral administration of $\gamma$-glutamylcysteine increases intracellular glutathione levels above homeostasis in a randomised human trial pilot study. Redox Biol 11: 631-636.

Zhou Q, Yan XF, Pan WS, and Zeng S (2008) Is the required therapeutic effect always achieved by racemic switch of proton-pump inhibitors? World J Gastroenterol 14:2617-2619.

Address correspondence to: Dr. Xiaoyan Chen, Shanghai Institute of Materia Medica, Chinese Academy of Sciences, 501 Haike Road, Shanghai 201203, China. E-mail: xychen@simm.ac.cn 